\title{
Improved Properties of PTFE Composites Filled with Glass Fiber Modified by Sol-Gel Method
}

Qiuying Li ( $\sim$ liqy@ecust.edu.cn )

East China University of Science and Technology https://orcid.org/0000-0003-1990-2402

Pengying Liu

East-China Institute of Technology: East China University of Science and Technology

Kashif Mahmood

East-China Institute of Technology: East China University of Science and Technology

Ning Zhang

East-China Institute of Technology: East China University of Science and Technology

Yanchao Che

Shanxi Funuo New Material Technology Co.,Ltd co.,

\section{Research Article}

Keywords: PTFE, GF, sol-gel, dielectric property, printed circuit board

Posted Date: March 16th, 2021

DOI: https://doi.org/10.21203/rs.3.rs-221609/v1

License: (c) (i) This work is licensed under a Creative Commons Attribution 4.0 International License.

Read Full License 


\section{Abstract}

Tetraethyl orthosilicate (TEOS) was used as the silicon source, and polydimethylsiloxane (PDMS) was the organic precursor to modify the surface of glass fiber (GF) through the sol-gel method. The modified GF noted T-GF was filled in PTFE to prepare PTFE/T-GF composites. SEM, FTIR, XPS, and contact angle confirmed that organic-inorganic hybrids were successfully loaded on GF's surface. Compared with PTFE/GF and the conventional coupling agent modified GF filled PTFE composites, the properties of PTFE/T-GF composites, including dielectric properties, mechanical properties, moisture absorption, thermal conductivity, and coefficient of thermal expansion (CTE), enhanced significantly for the improvement of compatibility of PTFE and GF. Moreover, the PTFE/T-GF exhibited improved dielectric constant (2.305), decreased dielectric loss $\left(9.08 \mathrm{E}^{-4}\right)$, higher bending strength $(21.45 \mathrm{MPa})$ and bending modulus $(522 \mathrm{MPa})$, better thermal conductivity $(0.268 \mathrm{~W} / \mathrm{m} * \mathrm{~K})$ and lower $\mathrm{CTE}\left(70 \mathrm{ppm} /{ }^{\circ} \mathrm{C}\right)$, making it has promising application as the substrate materials for printed circuit board.

\section{Introduction}

The rapid development of $5 \mathrm{G}$ communication technology requires the printed circuit board (PCB) substrate as the carrier to transmit high-speed signal should possess stable dielectric constant and low loss in the high-frequency range [1]. Polytetrafluoroethylene (PTFE) has become one of the most competitive materials for PCB substrate in high frequency as it is the polymer with the best dielectric properties due to its symmetric molecular structure and extremely low polarizability [2]. However, the disadvantages of PTFE, such as the high linear coefficient of thermal expansion (CTE $109 \mathrm{ppm} /{ }^{\circ} \mathrm{C}$ ) and poor creep resistance, limit its applications [3]. An efficient solution is to add glass fiber (GF) to the PTFE matrix. Alkali-free glass fiber (E-GF) with low permittivity, dielectric loss, and CTE $\left(0.5 \mathrm{ppm} /{ }^{\circ} \mathrm{C}\right)$ has been the ideal filler of high-performance, low dielectric PCB substrate [4-7].

However, the surface characteristics between PTFE and GF are incredibly different. PTFE has low surface energy and is hydrophobic, while GF has higher surface energy and shows hydrophilic. Therefore, the two interfaces are incompatible, raising the porosity and further impacting the composites' dielectric and mechanical properties [8]. By summarizing previous research, it can be found that there are usually two ways to improve the compatibility of PTFE and inorganic filler. One is to make the interface between PTFE and inorganic filler form a physical chimeric connection by increasing inorganic fillers' surface roughness. Han et al. [9] introduced hollow silica microspheres with porous surfaces into PTFE, which improved mechanical chimerism and significantly enhanced the composites' dielectric properties. The second is to treat the surface of inorganic filler with coupling agent $[6,8,10-13]$ to make PTFE (SP 12.7) and inorganic filler have similar Solubility Parameter (SP), thereby increasing the wettability of PTFE on the surface of inorganic filler and improving the interfacial adhesion [14]. Yao et al. [15] used F8261 coupling agent to treat ceramic and GF's surface, made the water absorption of the composite reach $0.12 \%$, and the dielectric loss decreased by 0.0024 . Although the coupling agent can improve the interfacial bonding between PTFE and GF, the interface failure still happened when PTFE/GF composites were used at high temperature because of the significant difference in CTE between GF and PTFE. 
The sol-gel method [16-20] is a reliable method to coat organic-inorganic hybrid layers on the fiber surface to improve its compatibility with the polymer matrix. In our previous work [20], tetraethyl orthosilicate (TEOS) used as the silicon source connected the silane coupling agent KH550 and polypropylene PP-g-MAH (MPP) to modify the surface of PET fiber by sol-gel method. A layer of organicinorganic hybrid film was formed on the surface of PET fiber. Moreover, the modified PET fiber filled PP composites showed outstanding interfacial and mechanical properties. Zhu et al. [21] also used TEOS as the silicon source and GPTMS as the silane coupling agent to modify PBO fiber. The formed $\mathrm{SiO}_{2}$ nanocoating significantly improved the interface compatibility between PBO fiber and epoxy resin. Therefore, it may be useful to modify GF by forming a hybrid coating through the sol-gel method.

In this study, inorganic layer from the hydrolysis and condensation of TEOS were designed to connect with the polydimethylsiloxane (PDMS) molecular chains through condensation reaction, which formed an organic-inorganic hybrid film with flake structure on the surface of GF. In this way, not only is the surface roughness of GF increased, but also the improvement of SP on the GF surface is realized (PDMS has similar SP (14.9) to PTFE), which is expected to enhance the compatibility between the GF and the PTFE matrix and improve the interface and dielectric properties of the composites. Moreover, the coupling agents modified GF, and untreated GF filled PTFE composites were also prepared for comparative analysis. The effects of different surface modifications of GF on the interface and dielectric properties between the fiber and the matrix were systematically studied.

\section{Experimental}

\subsection{Materials}

The E-glass fibers (length $=0.15 \mathrm{~mm}$ ) were provided by the Jushi Group Co., Ltd (China). PTFE powders with 20-25 $\mu \mathrm{m}$ diameter were supplied by Daikin Co., Ltd (Japan). Other materials including $\mathrm{Y}^{-}$ aminopropyl triethoxysilane (KH550, Shanghai Xinbo Chemical, China), phenyltriethoxysilane (Z6124, Sahn Chemical, China), tridecafluorooctyl trimethoxysilane (F8261, Shanghai Merrill Chemical Technology, China), tetraethyl orthosilicate (TEOS, Beijing Enokai Technology, China), and hydroxylterminated polydimethylsiloxane (PDMS, Beijing Enokai Technology, China) were also used.

\subsection{Preparation}

\subsubsection{Surface modification of GF}

(1) Preparation process of modified GF by combination of TEOS and PDMS:

15 wt.\% TEOS and 7.5 wt.\% PDMS, mass ratio to GF, were dissolved in ethanol under stirring for $6 \mathrm{~h}$ at room temperature. Simultaneously, the ammonia was added slowly into the liquid mixture until the $\mathrm{pH}$ was adjusted to 9 . Then GF washed with hydrochloric acid was added into the modified solution under stirring for $12 \mathrm{~h}$. Finally, the modified GF noted as T-GF was obtained after drying in the oven at $50{ }^{\circ} \mathrm{C}$ for one day. The specific response is shown in Scheme 1. 
(2) Preparation of modified GF with the coupling agents:

For comparison, the conventional coupling agents modified GF was prepared.

K-GF modified with the mixture of $\mathrm{KH} 550$ and $\mathrm{Z} 6124$ was prepared by the following process. Firstly, at 1.2 wt.\% $\mathrm{KH} 550$ and $0.3 \mathrm{wt} . \% \mathrm{Z} 6124$, mass ratio to GF, were dissolved in ethanol and deionized water to obtain coupling agent solution, where the volume ratio of deionized water: coupling agent: anhydrous ethanol $=1: 1: 8$. Then coupling agents were hydrolyzed at $55^{\circ} \mathrm{C}$ for $1 \mathrm{~h}$, and the $\mathrm{pH}$ was maintained between 4 and 6 adjusted by acetic acid. Next, GF was added to the coupling agent solution and stirred continuously for $6 \mathrm{~h}$. Finally, the mixture was dried in an oven at $80^{\circ} \mathrm{C}$ for $12 \mathrm{~h}$.

F-GF modified with F8261 coupling agent was similarly prepared by the above method, and the amount of F8261 was fixed at 1.5 wt.\% of GF.

\subsubsection{Preparation of PTFE/GF composites}

The PTFE and modified GF were first mixed in the mass ratio of 92:8 in a high-speed mixer under the speed of $2000 \mathrm{r} / \mathrm{min}$ for $5 \mathrm{~min}$ with ethanol as solvent, then filtrated 300 mesh sieve and dried at $90{ }^{\circ} \mathrm{C}$ for $24 \mathrm{~h}$. A high-speed grinder was used to smash the dried dough and got the PTFE/GF composite powders. Later, the powders were cold pressed at $15 \mathrm{MPa}$ for $5 \mathrm{~min}$, after that, the shaped rectangular sheets were sintered for $2 \mathrm{~h}$ under $370{ }^{\circ} \mathrm{C}$ and finally PTFE/GF composites were obtained.

\subsection{Measurements}

The GF and PTFE/GF samples' morphologies were characterized by scanning electron microscopy (SEM) (Hitachi, S-4800, Japan).

Fourier transform infrared (FTIR) spectra of untreated GF and modified GF were performed on Nicolet 6700 FTIR.

X-ray photoelectron (XPS) was used to analyze GF's surface characteristics on an ESCALAB 250Xi x-ray electron spectroscope (Thermo Fisher Co., USA) equipped with Al-Ka radiation as X-ray source.

The GF's contact angle was measured by the HARKE-SPACA contact angle measuring instrument according to the sessile drop method.

The dielectric constant and dielectric loss of the PTFE/GF composites were measured by the Agilent E8362B network analyzer using the strip-line resonance method. These dielectric properties were tested at the resonant frequency of $10 \mathrm{GHz}$.

PTFE/GF composites' bending properties were conducted by the three-point bending method on the universal mechanical machine (CMT4024-20KN, Shenzhen Sans Co., China). At least five specimens were tested for each type of composite.

The water absorption of the composites was measured according to IPC-TM-650 2.6.2. 
The thermal conductivity was measured by the laser flash method (Netzsch, LFA447, Germany) on circular specimens.

The CTE was tested according to the standard of IPC-TM-650. Before testing, the samples were immersed in isopropyl alcohol and mixed for $20 \mathrm{~s}$, then dried at $110{ }^{\circ} \mathrm{C}$ for $1 \mathrm{~h}$ and cooled to room temperature.

\section{Results And Discussion}

\subsection{Characterization of surface modification}

Figure 1 shows the microscopic morphology of modified GF and GF. As shown in Fig. 1 (a), GF's surface is relatively smooth except for some GF debris. However, after modified by silane coupling agents, some flaky protrusions can be observed faintly in Fig. 1 (b) and Fig. 1 (c) (marked with white arrows), which is the result of the coating of silane coupling. Unlike the coupling agents modified GF, T-GF's surface (Fig. 1 (d)) is occupied with a layer of flakes, and the surface roughness increased. Referring to the reaction mechanism in Scheme 1, the coating layer is organic and inorganic hybrid compounds formed in the hydrolytic condensation of TEOS and PDMS.

The surface modification of GF can be verified by detecting functional groups on its surface by FTIR, as presented in Fig. 2. Compared to GF, the T-GF infrared spectrum appears three new peaks, the peak at $2962 \mathrm{~cm}^{-1}$ is attributed to the stretching vibration of $\mathrm{C}-\mathrm{H}$ of TEOS and PDMS. The absorption bands at $1259 \mathrm{~cm}^{-1}$ and $800 \mathrm{~cm}^{-1}$ correspond to the $\mathrm{C}-0$ bond and Si-C bond's stretching vibration peak produced by TEOS and PDMS, respectively, proving that TEOS and PDMS have been loaded the surface of GF. In addition, the absorption peak at $1000-1100 \mathrm{~cm}^{-1}$ enhances (corresponding to the stretching vibration peak of Si-O bond), which is attributed to the hydrolysis condensation of TEOS and GF, supporting the reaction mechanism presented in Scheme 1.

In the spectrum of F-GF, two prominent characteristic peaks emerge at $2962 \mathrm{~cm}^{-1}$ and $2926 \mathrm{~cm}^{-1}$, pertaining to $\mathrm{CH}_{3}$ and $\mathrm{CH}_{2}$ anti-symmetric stretching vibration, respectively, which originate from F8261. This means that GF loaded with F8261 silane coupling agent has been successfully manufactured. In addition, an absorption peak of medium strength that appears at $1397 \mathrm{~cm}^{-1}$ is associated with the carboxyl group's stretching vibration peak, as the process of F8261 modified GF was catalyzed by acetic acid.

From K-GF, the stretching vibration absorption peaks of $\mathrm{CH}_{3}$ and $\mathrm{CH}_{2}$, which also appear at $2962 \mathrm{~cm}^{-1}$ and $2926 \mathrm{~cm}^{-1}$, are characteristic absorption peaks from KH550. Also, an absorption peak appears at $3100-3300 \mathrm{~cm}^{-1}$, attributed to the stretching vibration peak of $\mathrm{NH}_{2}$ in $\mathrm{KH} 550$, verifying that $\mathrm{KH} 550$ has been connected to the GF surface. The absorption peak at $1557 \mathrm{~cm}^{-1}$ corresponds to the stretching vibration peak of $\mathrm{C}=\mathrm{C}$ of the benzene ring, and the double peaks at $737 \mathrm{~cm}^{-1}$ and $700 \mathrm{~cm}^{-1}$ are the outof-plane bending peak of the unitary substituted $\mathrm{C}-\mathrm{H}$ bond in the benzene ring, which confirms that Z6124 
containing the characteristic group-benzene ring has been successfully loaded on the GF surface. Similarly, the characteristic peak at $1397 \mathrm{~cm}^{-1}$ is the characteristic peak of acetic acid.

XPS provides information on molecular structure and valence state, and also provides information on element composition and content of various materials for chemical research further to analyze the surface-modified effect of GF through XPS. Figure 3 shows the XPS spectra of F-GF, T-GF, and K-GF. It could be seen that the F-GF appears a pronounced F1s peak, indicating that F8261 has been successfully loaded on the GF surface. The peak of N1s appears in the XPS spectrum of K-GF, which confirms that KH550 has been connected to GF's surface.

Fig. 4 depicts the Si2p XPS spectra of GF and T-GF. As shown from the spectra, in addition to the fitting peaks at $103.2 \mathrm{eV}$ and $102.36 \mathrm{eV}$ (corresponding to the binding energy of silicon elements in $\mathrm{SiO} 2$ and CaSiO3), the surface of T-GF also shows the $\mathrm{Si}-\mathrm{O}$ bond and $\mathrm{Si}-\mathrm{C}$ bond, corresponding to the binding energy of $101.2 \mathrm{eV}$ and $100.8 \mathrm{eV}$ respectively. The Si-O bond was formed by the hydrolytic condensation reaction of TEOS, while the Si-C bond was formed by the dehydration condensation of PDMS and TEOS, thus further illustrating that the hydrolytic condensation reaction of TEOS and PDMS has taken place successfully.

Their SP influences the compatibility of matrix and filler in the composite material and fundamentally depends on the polarity of functional groups of the materials, characterized by surface energy. The water contact angle can reflect the material's surface energy, so the compatibility of modified GF with PTFE could be judged by measuring the modified GF's water contact angle. The surface energy of PTFE is deficient at $18.5 \mathrm{mN} / \mathrm{m}$. According to Young's formula [22], the larger the water contact angle of a material, the lower its surface energy, and the better its compatibility with PTFE. The contact angles of KGF, F-GF, and T-GF are displayed in Fig. 5. The unmodified GF is fully water-absorbing. But after surface modification, GF changed from hydroscopic to hydrophobic, and the contact angles are greater than $100^{\circ}$. T-GF displays the largest contact angle, reaching $117^{\circ}$, attributed to the low surface energy of PDMS and the modified rough surface. The contact angle of K-GF is smaller, mainly due to the larger polarity of amino in the coupling agent that increases the material's surface energy.

\subsection{The microstructure and properties of PTFE/GF composites}

The interface is an important factor affecting the properties of composite materials. The two-phase interface in PTFE/GF could be observed in Fig. 6 . Figure 6 (a) shows that the interface adhesion between GF and PTFE matrix is weak for apparent gaps appear on the interface, resulting from the large surface energy difference between PTFE and GF. As shown in Fig. 6 (b) and (c), although the coupling agent treatment reduces GF and PTFE gaps to a certain extent, the modification effect is still limited. Figure 6 (d) shows that the interface gaps between T-GF and PTFE almost disappear, and the surface of the T-GF and PTFE matrix form a better interface connection. This could be attributed that the inorganic-organic hybrid coating formed by the hydrolysis and condensation of TEOS and PDMS can increase the surface roughness of GF (as shown in Fig. 2), and the PDMS organic polymer grafted on the surface of GF can 
form molecular chains entanglement with PTFE, making it form a compact interface structure and firm connection with PTFE.

The dielectric constant of the PCB substrate materials determines the signal transmission rate, and the dielectric loss affects the signal attenuation and is the key factor to produce heat loss. Therefore, the dielectric constant and dielectric loss are the essential performance characteristics of the PCB substrate material. Figure 7 (a) shows the dielectric constant and dielectric loss of the PTFE/GF composites at 10 GHz. PTFE/GF, PTFE/T-GF and PTFE/K-GF display slightly lower dielectric constant and higher dielectric loss than PTFE/T-GF. As described above, the interface connection between GF, K-GF and F-GF and PTFE is weak. The air in the interfacial gap will reduce the dielectric constant of the material. The high dielectric performance of PTFE/T-GF are due to the organic-inorganic hybrid grafted on the surface of GF, which makes GF and PTFE have a good affinity, resulting in a strong interface connection and a dense interface structure of the PTFE/T-GF composite materials, thereby improved electrical properties. Otherwise, the gaps between two phases will lead to additional interface polarization, causing electrons or ions in the dielectric to gather at the interface, resulting in the increase of dielectric loss. Which is the main reason that the dielectric loss of PTFE/T-GF is lower $\left(\tan \delta=9.08 \mathrm{E}^{-4}\right)$ than those of untreated, and the coupling agents modified GF filled PTFE.

The dielectric properties of the composites are essentially the result of each component's dielectric properties and the interaction of the interface properties formed between them. Many theories have been used to predict the dielectric constant of the composite materials [23-26], the equations of these models were enclosed as following:

1. Effective medium theory (EMT) [24]

$$
\varepsilon_{c}=\varepsilon_{1}\left[1+\frac{V_{2}\left(\varepsilon_{2}-\varepsilon_{1}\right)}{\varepsilon_{1}+m\left(1+V_{2}\right)\left(\varepsilon_{2}-\varepsilon_{1}\right)}\right]
$$

2. Modified Lichtenecker equation [26]

$$
\log \varepsilon_{c}=V_{2}(1-n) \log \left(\frac{\varepsilon_{2}}{\varepsilon_{1}}\right)+\log \varepsilon_{1}
$$

3. Maxwell-Wagner equation [23]

$$
\varepsilon_{c}=\varepsilon_{1} \frac{2 \varepsilon_{1}+\varepsilon_{2}+2 V_{2}\left(\varepsilon_{2}-\varepsilon_{1}\right)}{2 \varepsilon_{1}+\varepsilon_{2}-V_{2}\left(\varepsilon_{2}-\varepsilon_{1}\right)}
$$


Where $\varepsilon_{\mathrm{c}}, \varepsilon_{1}$, and $\varepsilon_{2}$ represents the dielectric constant of the composites, the matrix and fillers, respectively, $V_{2}$ is the fillers' volume fraction, $m$ in Eq. (1) relates to a morphology factor, while $n$ in Eq. (2) is a fitting parameter. Further, the parameter $\mathrm{m}$ in this paper is set to 0.17 , which is the same as the PTFE/CNT composites reported by Lin [27]. Besides, the Modified Lichtenecker model matches well with experimental data when factor $\mathrm{n}$ is fixed at 0.28 . Figure 7 (b) shows the comparison of experimental permittivities and theoretical dielectric constant predicted by above mentioned models. Obviously, the dielectric change trend of PTFE/T-GF with the increase of GF content is similar to the theoretical calculation value. Moreover, the modified Lichtenecker and EMT model fit well with the experiments, the percentage deviation is kept below $1 \%$. Indicating that PTFE and T-GF in composites are closely bound to each other, which is consistent with the two-phase structure of the application conditions of the formulas, and confirming that the experimental data of dielectric constant is credible and predictable. However, the results calculated by the Maxwell-Wagner model are lower than the experimental values. In many previous studies [12], Maxwell-Wagner model was only proved to be available at low working frequency, therefore, the high frequency test condition at $10 \mathrm{GHz}$ is the main reason for the deviation. Otherwise, with the increase of GF filler content, the dielectric of PTFE/GF composites gradually deviates from the theoretical calculation value, which is attributed to the weak interface connection between GF and PTFE. Consequently, it further proves that the interfacial binding property of PTFE and GF plays a vital role in the composite materials' dielectric properties.

The bending strength and modulus are two important mechanical indicators of PCB substrate materials. The PCB plays a role in supporting circuit components, so bending resistance requirements are put forward [28]. The bending strength and modulus of PTFE/GF composites are represented in Fig. 8. The results show that the bending strength and modulus of the composites increase with enhancing the interface connection between GF and PTFE. So, the bending strength and bending modulus of PTFE/T-GF are the highest, $21.45 \mathrm{MPa}$ and $522 \mathrm{MPa}$, respectively, $8 \%$ and $13 \%$ higher than those of PTFE/GF. The inorganic and organic hybrid layers coated on the surface of T-GF act as the interface transition layer between PTFE and GF to play the role of stress relaxation. When the composite material is stressed, PTFE can transfer the stress to GF through the transition layer, resulting in increasing the composite strength.

The application environment of the circuit board is complex and changeable, especially in a humid environment. To keep the dielectric properties of the dielectric substrate, it is necessary to ensure it has low water absorption. According to previous studies [4, 6], the water absorption of PTFE/GF composites is since GF's surface contains a large number of hydrophilic groups-hydroxyl, which can capture water molecules to form bound water. It also produces greater water absorption due to its low compatibility with PTFE; the porosity thus allows water to penetrate the composite material's interior. Therefore, it is highly significant to perform surface treatment on GF to form a hydrophobic coating on the surface and improve its compatibility with PTFE. Table 1 shows the effects of different surface treatment methods on the density, porosity and water absorption of the composites. The densities of GF and PTFE polymer are 2.76 and $2.15 \mathrm{~g} / \mathrm{cm}^{3}$, respectively. Generally, the theoretical density $\left(\rho_{0}\right)$ was calculated by Eq. (4) and determined to be 2.188 . The porosity was calculated by Eq. (5). 


$$
\begin{gathered}
\rho_{0}=\rho_{1} V_{1}+\rho_{2} V_{2} \\
\rho_{r}=\frac{\rho}{\rho_{0}}
\end{gathered}
$$

Where $\rho_{1}, \rho_{2}$ represents the density of the PTFE and GF fillers, respectively, $V_{1}$ and $V_{2}$ represent the matrix and fillers' volume fraction. As shown in Table 1, GF's surface treatment with the coupling agent significantly reduces the porosity and water absorption of the composite material. Moreover, the use of sol-gel modification can reduce the water absorption $(0.0167 \%)$ to a greater extent. The porosity and water absorption of composites mainly depends on the interface compatibility between the polymer and fillers. Using the sol-gel method to coat the surface of PTFE with a layer of inorganic and organic hybrids could enhance the interface connected, thereby obtaining a lower water absorption. Meanwhile, all the above results can be confirmed from the microstructure of all the composites shown in Fig. 6.

Table 1

Density, porosity and water absorption of PTFE/GF composites

\begin{tabular}{|lllll|}
\hline Sample & PTFE/GF & PTFE/K-GF & PTFE/F-GF & PTFE/T-GF \\
\hline Density $\left(\mathrm{g} / \mathrm{cm}^{3}\right)$ & 2.123 & 2.144 & 2.141 & 2.178 \\
\hline Porosity (\%) & 0.030 & 0.021 & 0.022 & 0.005 \\
\hline Water absorption (\%) & 0.018 & 0.0174 & 0.0176 & 0.0167 \\
\hline
\end{tabular}

The heating of the PCB often leads to a decrease in the substrate material's dielectric performance, thereby affecting signal transmission. Therefore, a suitable thermal conductivity is also an essential prerequisite for ensuring the PCB substrate's reliability. The thermal conductivity of PTFE/GF composites are shown in Fig. 9 (a). As could be seen, the thermal conductivity of PTFE/T-GF is significantly enhanced, reaching $0.268 \mathrm{~W} / \mathrm{m} * \mathrm{~K}$, which is $23 \%$ higher than that of PTFE/GF. Meanwhile, the thermal conductivities of PTFE/K-GF and PTFE/F-GF are also slightly improved. The reason lies in the interface between the filler, and the matrix will cause the scattering of phonons. The larger the interface pores, the greater the scattering loss, and the worse the thermal conductivity $[5,29]$. In contrast, the surface modifier acts as a bridge between GF and PTFE to improve the composite material's thermal conductivity. The effect of different filler content on the thermal conductivity is shown in Fig. 9 (b), but it is found that the filler content has little effect on the thermal conductivity of the composite material, which may be due to the lower filler content being insufficient to form a thermal conduction channel. Therefore, the influence on the thermal conductivity of the interface properties of the material is much larger than the content of the filler (filler content in the case where the thermal conductivity is not more than the threshold value) on the thermal conductivity properties of the PTFE/GF composites.

For the sake of preventing the copper conductor path of PCB from breaking during the heating process, the CTE of the substrate should match that of the copper foil $\left(18 \mathrm{ppm} /{ }^{\circ} \mathrm{C}\right)$ as much as possible. The 
thermal expansion curve of PTFE and GF reinforced composites is shown in Fig. 10 (a). The slope of the curve represents the CTE. The average CTE of PTFE and GF reinforced composites at $25-100{ }^{\circ} \mathrm{C}$ is shown in Fig. 10 (b). The results show that the addition of GF filler can significantly reduce the CTE of the material. This is because GF dispersed in the PTFE matrix acts as a motion barrier to limit the thermal expansion of PTFE. Moreover, the CTE of composites decreases with the enhancement of the interface bonding force between GF and PTFE. At last, PTFE/T-GF maintains the lowest CTE, at $70 \mathrm{ppm} /{ }^{\circ} \mathrm{C}$, which is $21 \%$ lower than that of PTFE/GF, indicating that the interface properties have a significant impact on the thermal properties of the composite.

The CTE of PTFE and its composite material changes with temperature (Fig. 10 (c) and (d)) is obtained by deriving deformation. As shown in Fig. 10 (c), it could be observed that when the temperature is lower than $260{ }^{\circ} \mathrm{C}$, the CTE of PTFE remains in a relatively stable range. When the temperature exceeds $260{ }^{\circ} \mathrm{C}$, its CTE increases exponentially. According to previous studies [30,31], the molecular chains of PTFE gradually changed from a regular crystalline state to a stretched amorphous structure with the temperature increasing. Thereby the applicable range of PTFE should be lower than $260^{\circ} \mathrm{C}$. The CTE curves within the range of 25 to $120^{\circ} \mathrm{C}$ are presented in Fig. 10 (d). The CTE of PTFE and its composite materials are maintained in a stable range. But at $30{ }^{\circ} \mathrm{C}$, the CTE of the PTFE composites increases significantly; because the PTFE crystals undergo crystalline relaxation at that temperature, causing the spiral of the chains to become irregularly entangled [32]. It is worth noting that the temperature point of the crystal relaxation is within the usual temperature range, resulting in the volume of PTFE changing significantly and accordingly generating a certain impact on the application properties of PTFE and its composite materials.

\section{Conclusion}

The sol-gel method was used successfully to construct an organic-inorganic hybrids coating on the surface of GF, and then the modified GF noted T-GF was filled into PTFE to obtain PTFE/T-GF composites, which showed better performance than PTFE composites filled with GF modified by coupling agents, with a dielectric constant of 2.305 , a dielectric loss of $9.08 \mathrm{E}^{-4}$, a bending strength of $21.45 \mathrm{MPa}$, and a bending modulus of $522 \mathrm{MPa}$, the moisture absorption of $0.0167 \%$, the thermal conductivity of 0.268 $\mathrm{W} / \mathrm{m}{ }^{\star} \mathrm{K}$, and the CTE of $70 \mathrm{ppm} /{ }^{\circ} \mathrm{C}$. The performance improvement of PTFE/T-GF composite material is attributed to the formation of an organic-inorganic hybrid layer on the surface of GF, which increases the surface roughness of GF and at the same time improves the SP on the surface of GF to make it more compatible with PTFE. The SEM micrograph of the composite material's cross-section shows that a dense interface structure is formed between T-GF and PTFE. The sol-gel surface modification provides a promising method to modify the surface of GF or other inorganic particles to improve the interface performance of composite materials and develops the application of PTFE-based PCB substrates.

\section{References}


1. H. Xu, W.J. Liang, Q.L. Gao, A new dual-channel measurement method for accurate characterization of low-permittivity and low-loss materials. IEEE Trans. Instrum. Meas. 67, 1370-1379 (2018)

2. Y. Yuan, D.D. Yu, Y.T. Yin, B. Tang, E.Z. Li, S.R. Zhang, Effect of sintering temperature on the crystallization behavior and properties of silica filled PTFE composites. J. Mater. Sci.-Mater. Electron. 27, 13288-13293 (2016)

3. K.P. Murali, S. Rajesh, K.J. Nijesh, R. Ratheesh, Effect of particle size on the microwave dielectric properties of alumina-filled PTFE substrates. International Journal of Applied Ceramic Technology. 7, 475-481 (2010)

4. F.K. Huang, Y. Yuan, Z.H. Jiang, B. Tang, S.R. Zhang, Microstructures and properties of glass fiber reinforced PTFE composite substrates with laminated construction. Mater. Res. Express. 6, 10 (2019)

5. Y. Zhang, K.C. Kou, S.C. Zhang, G.L. Wu, Effect of fiber diameter on thermal properties of short-glassfiber-reinforced PTFE-based composites. J. Mater. Sci.-Mater. Electron. 31, 10715-10723 (2020)

6. Z.H. Li, J.S. Liu, Y. Yuan, E.Z. Li, F. Wang, Effects of surface fluoride-functionalizing of glass fiber on the properties of PTFE/glass fiber microwave composites. RSC Adv. 7, 22810-22817 (2017)

7. Y. Yuan, J. Yang, B.L. Tan, B. Tang, E.Z. Li, S.R. Zhang, Preparation, characterization and properties of FEP modified PTFE/glass fiber composites for microwave circuit application. J. Mater. Sci.-Mater. Electron. 28, 6015-6021 (2017)

8. Y. Yuan, Z.F. Chi, B. Tang, E.Z. Li, S.R. Zhang, Research on hydrophobicity treatment of aluminum nitride powder and the fabrication and characterization of AIN/PTFE composite substrates. J. Mater. Sci.-Mater. Electron. 29, 14890-14896 (2018)

9. K.K. Han, J. Zhou, Q.Z. Li, J. Shen, Y.Y. Qi, X.P. Yao, W. Chen, Effect of filler structure on the dielectric and thermal properties of $\mathrm{SiO}_{2}$ /PTFE composites. J. Mater. Sci.-Mater. Electron. 31, 9196-9202 (2020)

10. F.C. Luo, B. Tang, Z.X. Fang, Y. Yuan, H. Li, S.R. Zhang, Effects of coupling agent on dielectric properties of PTFE based and $\mathrm{Li}_{2} \mathrm{Mg}_{3} \mathrm{TiO}_{6}$ filled composites. Ceram. Int. 45, 20458-20464 (2019)

11. Y. Yuan, Y.T. Yin, D.D. Yu, H.D. Lin, J. Wang, B. Tang, E.Z. Li, Effects of compound coupling agents on the properties of PTFE/ $\mathrm{SiO}_{2}$ microwave composites. J. Mater. Sci.-Mater. Electron. 28, 3356-3363 (2017)

12. F.C. Luo, B. Tang, Z.X. Fang, Y. Yuan, H. Li, S.R. Zhang, Polytetrafluoroethylene based, F8261 modified realization of $\mathrm{Li}_{2} \mathrm{SnMg}_{0.5} \mathrm{O}_{3.5}$ filled composites. Appl. Surf. Sci. 503, 7 (2020)

13. H. Wang, F.M. Zhou, J.M. Guo, H. Yang, J.X. Tong, Q.L. Zhang, Modified BCZN particles filled PTFE composites with high dielectric constant and low loss for microwave substrate applications. Ceram. Int. 46, 7531-7540 (2020)

14. M. Murase, R. Ohta, Prediction of molecular affinity on solid surfaces via three-dimensional solubility parameters using interfacial free energy as interaction threshold. J. Phys. Chem. C 123, 1324613252 (2019) 
15. M.H. Yao, Y. Yuan, E.Z. Li, B. Tang, S.R. Zhang, Effects of $(\mathrm{Na} 1 / 2 \mathrm{Nd} 1 / 2) \mathrm{TiO}_{3}$ on the microstructure and microwave dielectric properties of PTFE/ceramic composites. J. Mater. Sci.-Mater. Electron. 29, 20680-20687 (2018)

16. X.J. Su, H.Q. Li, X.J. Lai, L. Zhang, J. Wang, X.F. Liao, X.R. Zeng, Vapor-liquid sol-gel approach to fabricating highly durable and robust superhydrophobic polydimethylsiloxane@silica surface on polyester textile for oil-water separation. ACS Appl. Mater. Interfaces. 9, 28089-28099 (2017)

17. C. Kapridaki, A. Verganelaki, P. Dimitriadou, P. Maravelaki-Kalaitzaki, Conservation of monuments by a three-layered compatible treatment of TEOS-nano-calcium oxalate consolidant and TEOS-PDMS$\mathrm{TiO}_{2}$ hydrophobic/photoactive hybrid nanomaterials. Materials. 11, 684-706 (2018)

18. C. Rosu, H.S. Lin, L. Jiang, V. Breedveld, D.W. Hess, Sustainable and long-time 'rejuvenation' of biomimetic water-repellent silica coating on polyester fabrics induced by rough mechanical abrasion. J. Colloid Interface Sci. 516, 202-214 (2018)

19. A.C. Pierre, D.R. Uhlmann, A. Hordonneau, Ceramic composites made by sol-gel processing. Revue Internationale des Hautes Temperatures et des Refractaires. 23, 29-35 (1986)

20. Y.P. Mao, Q.Y. Li, C.F. Wu, Surface modification of pet fiber with hybrid coating and its effect on the properties of PP composites. Polymers. 11, 1726-1740 (2019)

21. C.H. Zhu, B. Wang, J.F. Meng, S.Q. Deng, X.N. Fan, T. Peng, W.L. Zhou, Y.T. Li, H. Li, C.X. Zhao, Surface modification of $\mathrm{PBO}$ fiber by grafting nanoporous $\mathrm{SiO}_{2}$ coating with improved interfacial properties. Mater. Res. Express. 6, 9 (2019)

22. A.S. Almusallam, Effect of interfacial curvature on the adsorption of copolymer stabilized nanoparticles of different copolymer compositions: a Brownian dynamics study. Phys. Chem. Chem. Phys. 12, 12198-12207 (2010)

23. Y.Y. Sun, Z.Q. Zhang, C.P. Wong, Influence of interphase and moisture on the dielectric spectroscopy of epoxy/silica composites. Polymer. 46, 2297-2305 (2005)

24. Y. Rao, J.M. Qu, T. Marinis, C.P. Wong, A precise numerical prediction of effective dielectric constant for polymer-ceramic composite based on effective-medium theory. IEEE Trans. Compon. Packaging Technol. 23, 680-683 (2000)

25. J. Krupka, S.A. Gabelich, K. Derzakowski, B.M. Pierce, Comparison of split post dielectric resonator and ferrite disc resonator techniques for microwave permittivity measurements of polycrystalline yttrium iron garnet. Meas. Sci. Technol. 10, 1004-1008 (1999)

26. A.H. Sihvola, J.A. Kong, Effective permittivity of dielectric mixtures. IEEE Transactions on Geoscience and Remote Sensing. 26, 420-429 (1988)

27. P. Haiyi, R. Haishen, D. Mingzhao, Novel high dielectric constant and low loss PTFE/CNT composites. Ceramics International. 44, 16556-16560 (2018)

28. H. Wang, H. Yang, J.X. Tong, Q.L. Zhang, Medium dielectric constant and low-loss PTFE composites filled with MgO-LiF co-doped $\mathrm{Li}_{2} \mathrm{TiO}_{3}$ particles. J. Appl. Polym. Sci. 136, 9 (2019) 
29. C. Pan, K.C. Kou, Y. Zhang, Z.Y. Li, T.Z. Ji, G.L. Wu, Investigation of the dielectric and thermal conductive properties of core-shell structured HGM@hBN/PTFE composites. Mater. Sci. Eng. B-Adv. Funct. Solid-State Mater. 238, 61-70 (2018)

30. S.A. Serov, S.A. Khatipov, N.V. Sadovskaya, A.V. Tereshenkov, N.A. Chukov, Double melting in polytetrafluoroethylene gamma-irradiated above its melting point. Nuclear Instruments \& Methods in Physics Research Section B-Beam Interactions with Materials and Atoms. 271, 92-95 (2012)

31. Y. Seo, Nonisothermal crystallization kinetics of polytetrafluoroethylene. Polymer Engineering and Science. 40, 1293-1297 (2000)

32. Z.-C. Wang, K.-C. Kou, M. Chao, H. Bi, L.-K. Yan, Nonisothermal crystallization kinetics of polytetrafluoroethylene/solid glass microsphere composites. J. Appl. Polym. Sci. 117, 1218-1226 (2010)

\section{Figures}

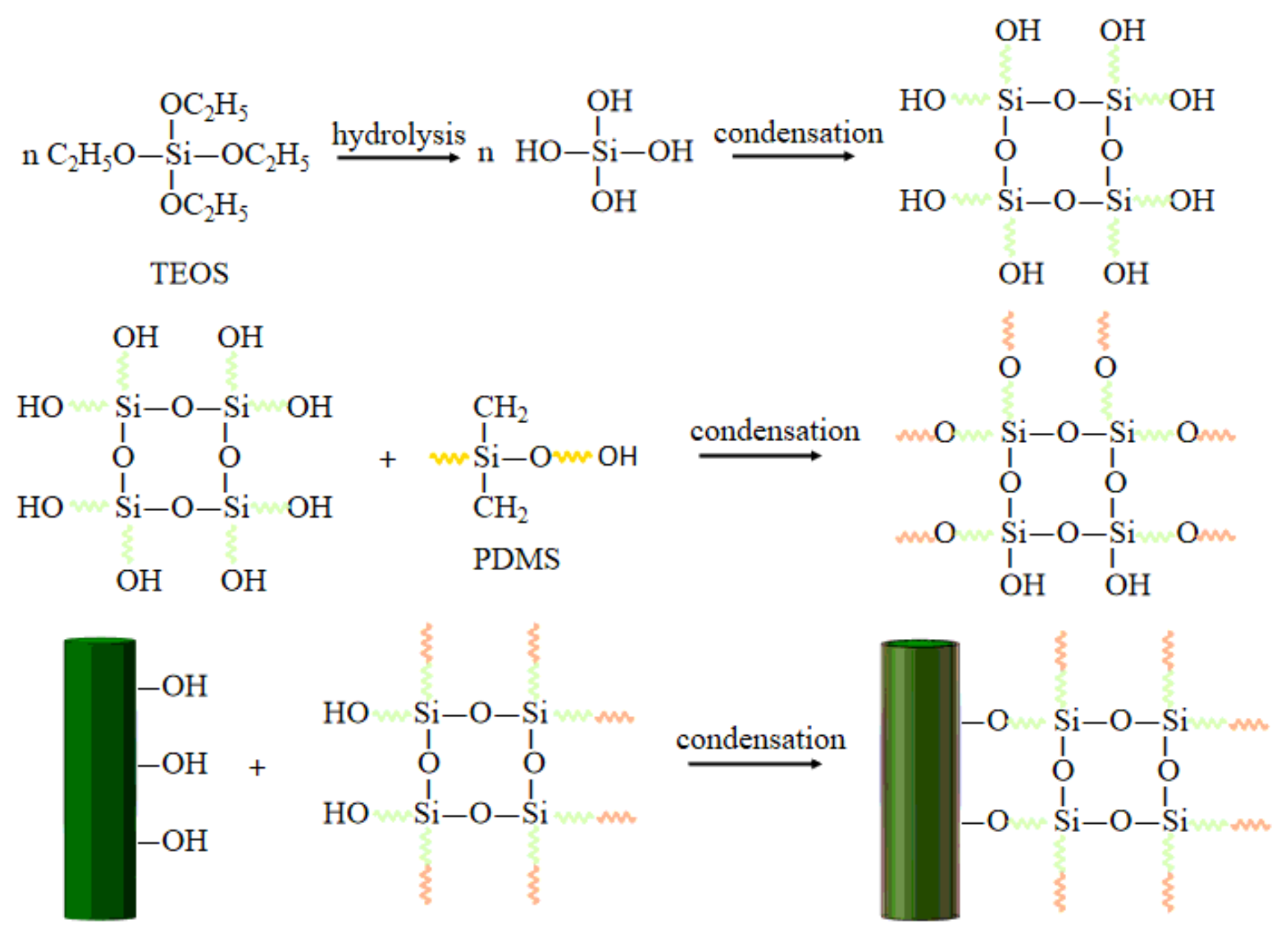

Figure 1 
Schematic diagram of TEOS, PDMS, and GF reactions
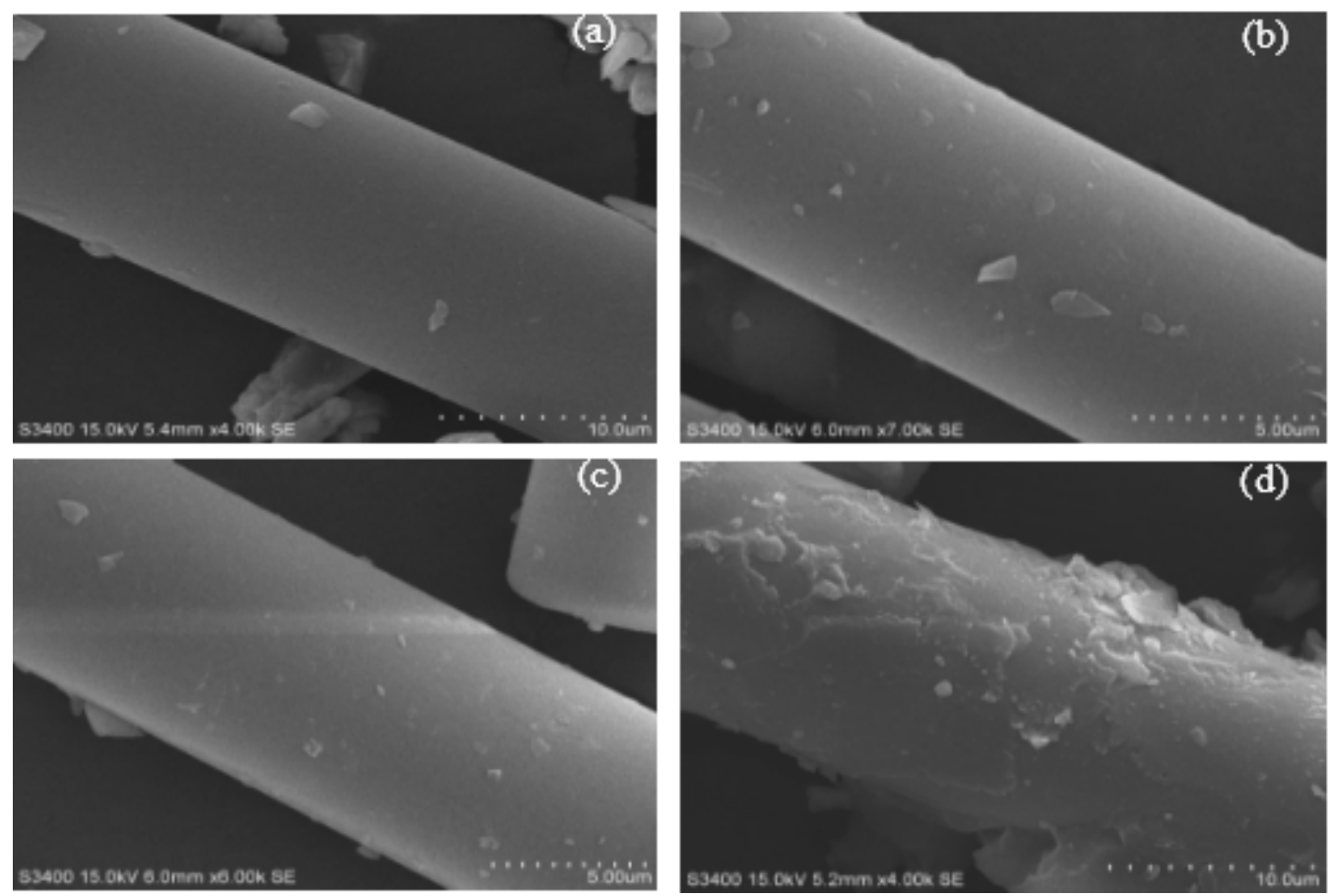

Figure 2

SEM images of the surfaces of (a) GF, (b) K-GF, (c) F-GF, and (d) T-GF

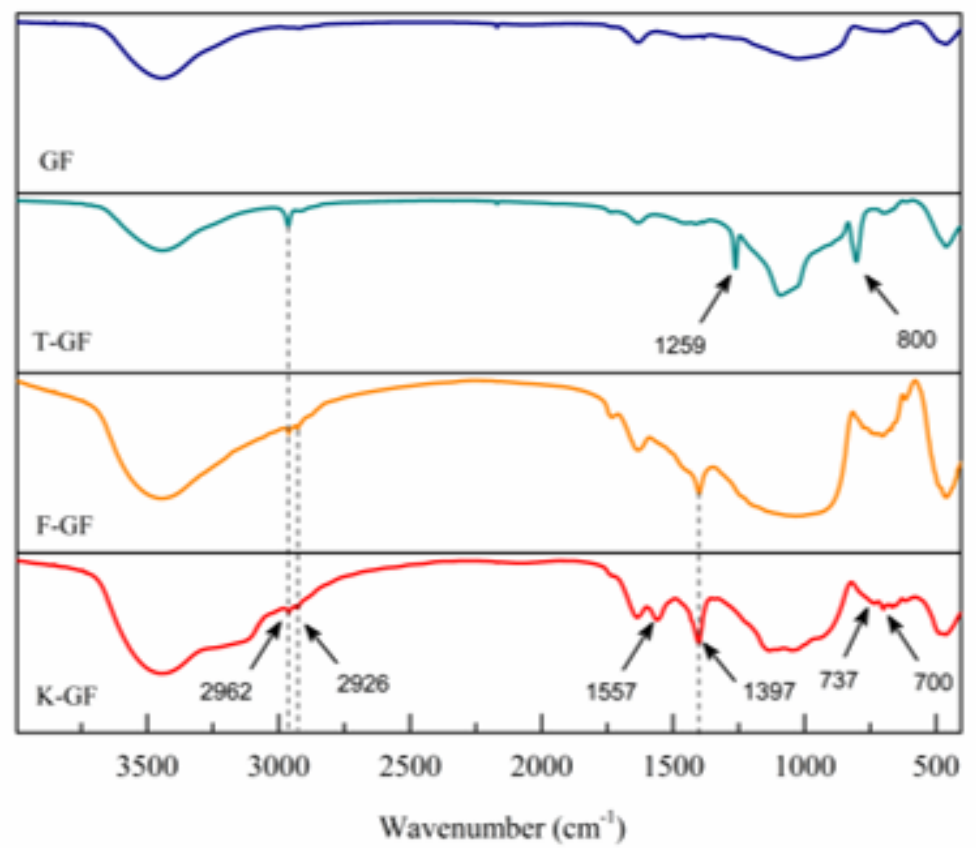

Figure 3

FTIR spectra of GF and modified GF 


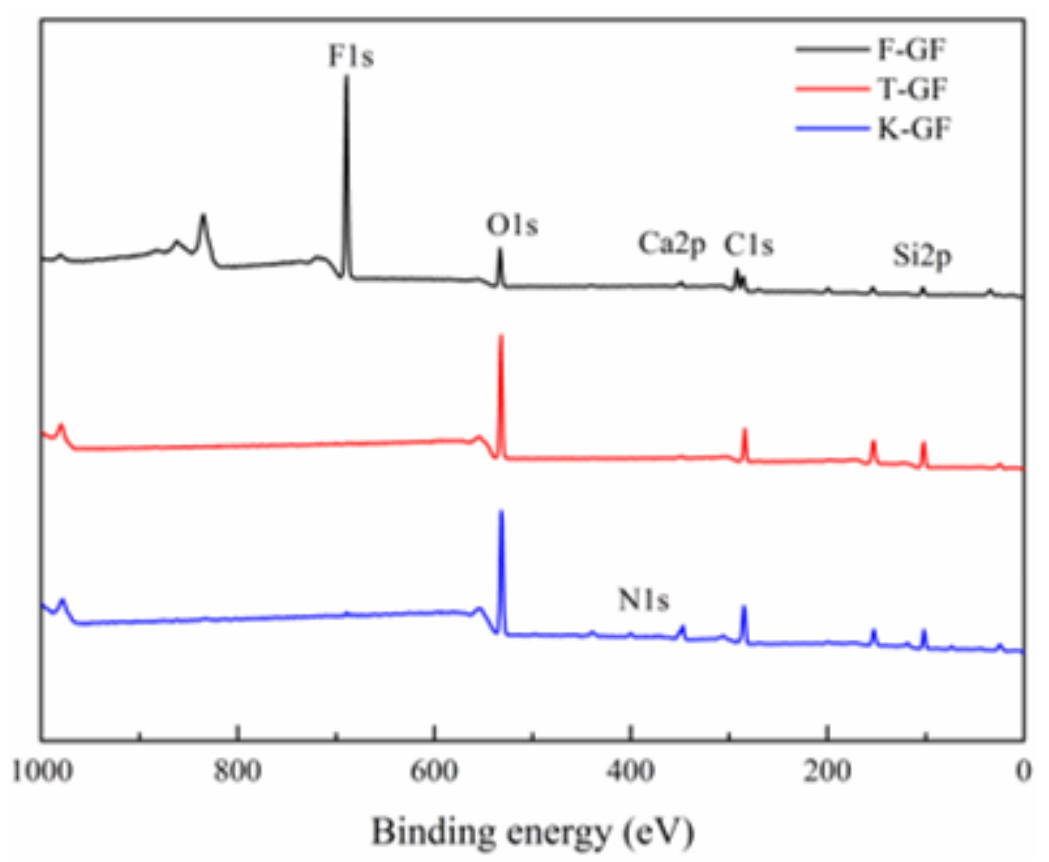

Figure 4

XPS spectra of F-GF, T-GF, and K-GF
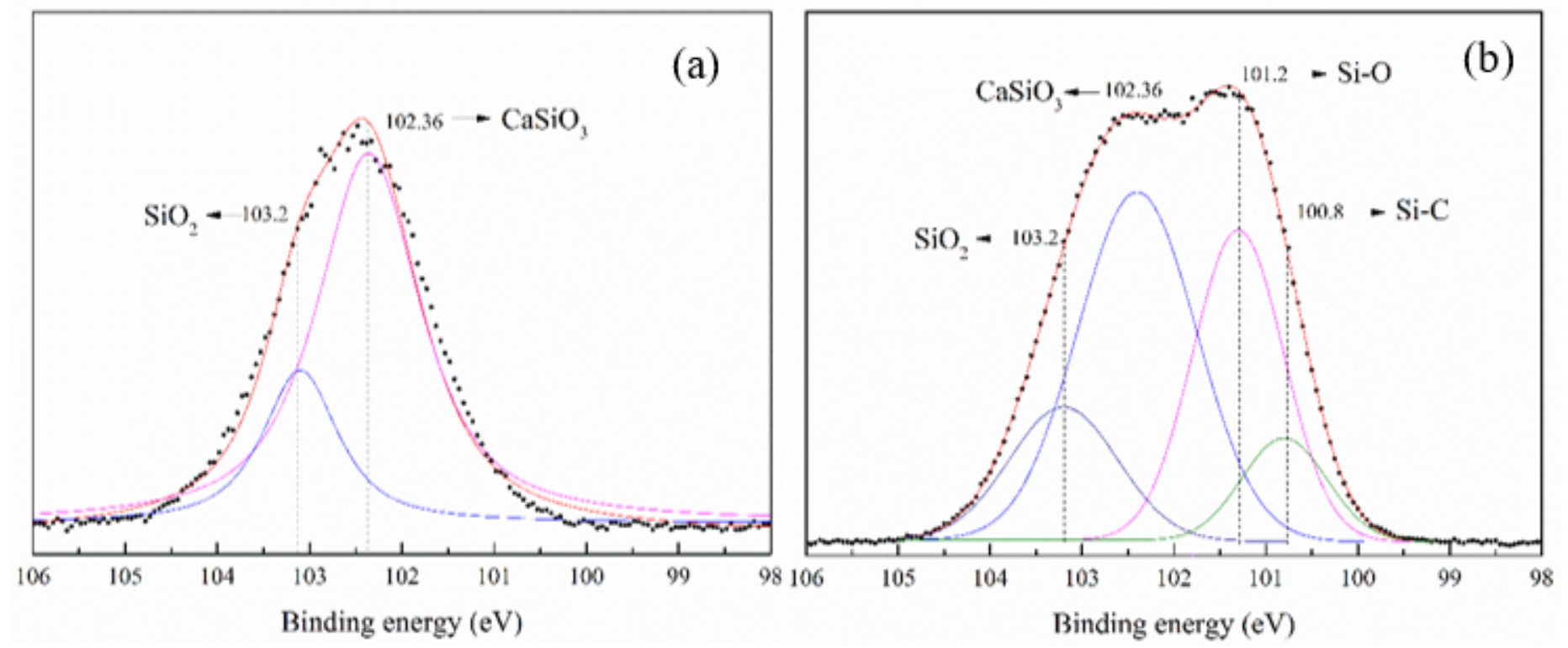

\section{Figure 5}

Si2p XPS spectra of (a) GF and (b) T-GF 


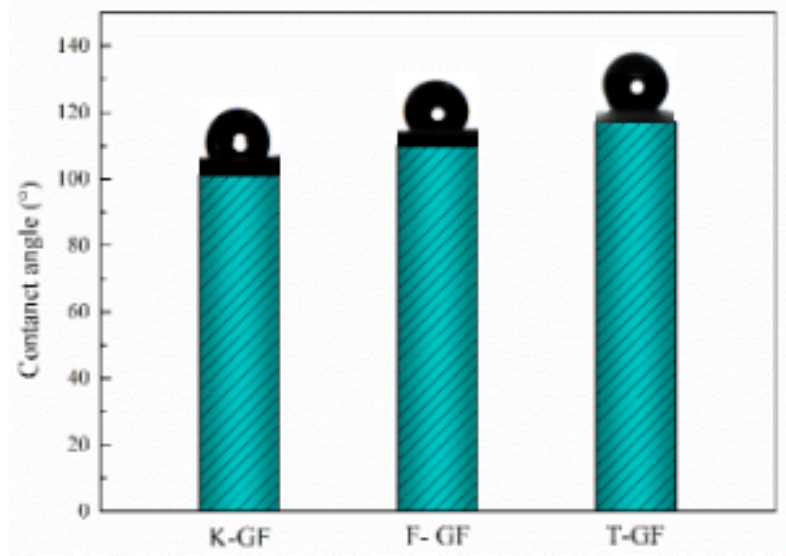

Figure 6

Contact angles of K-GF, F-GF and T-GF
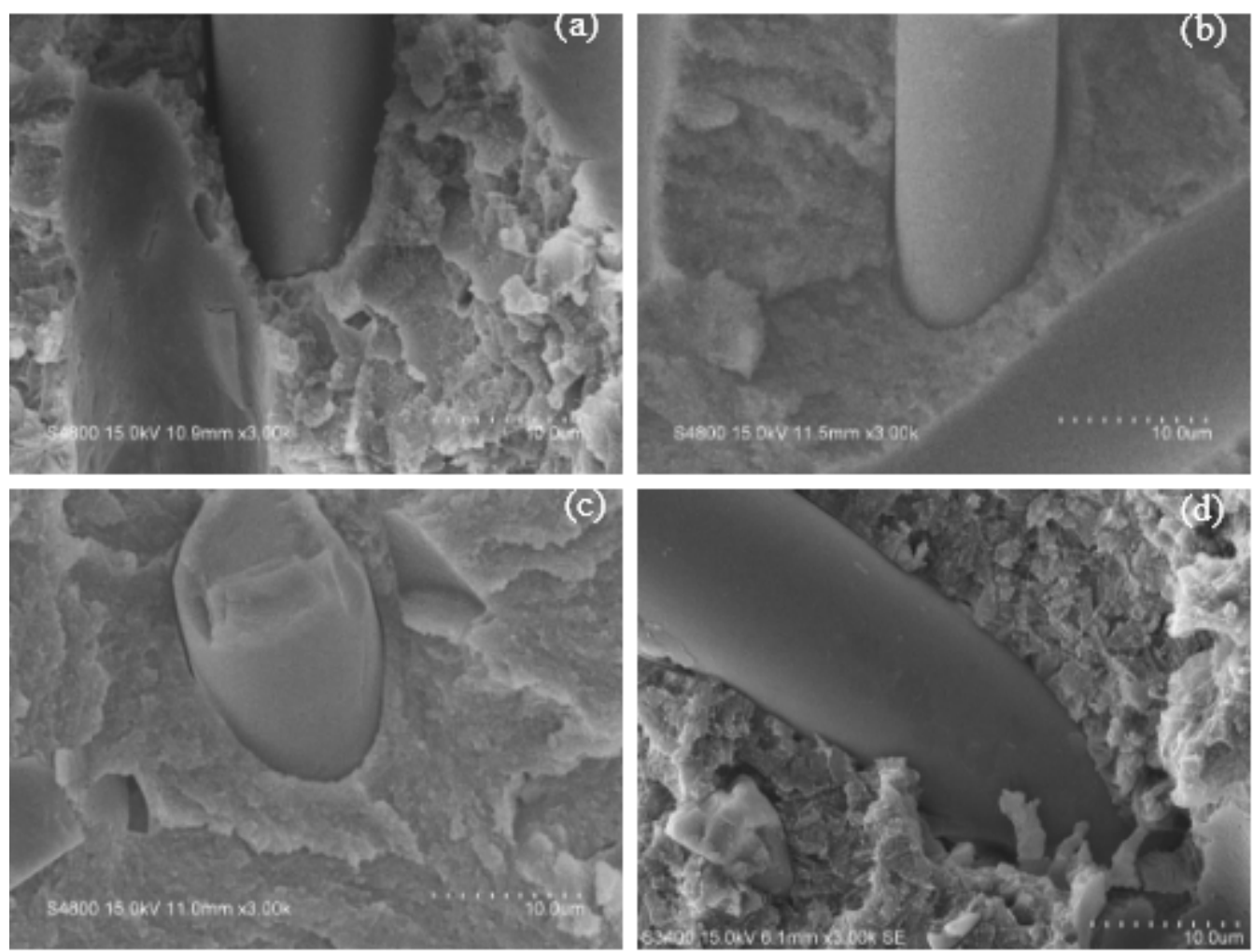

Figure 7

SEM images of cross-section of (a) PTFE/GF, (b) PTFE/K-GF, (c) PTFE/F-GF and (d) PTFE/T-GF 

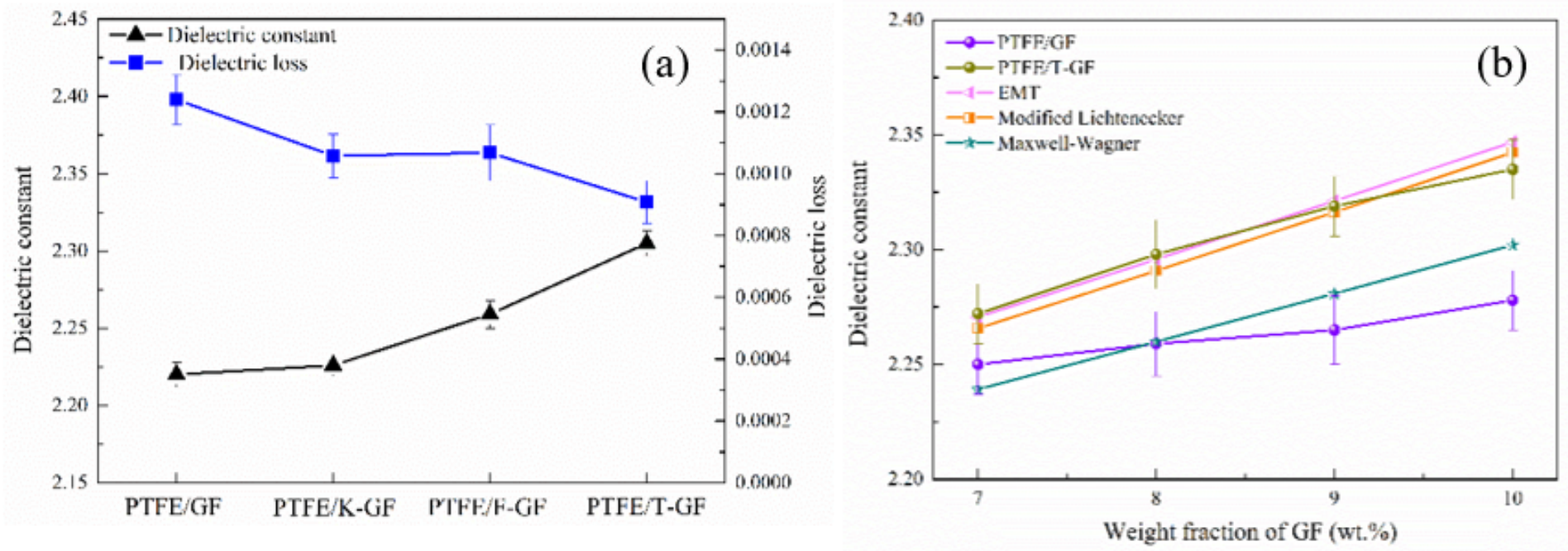

Figure 8

(a) Dielectric constant and dielectric loss of PTFE/GF composites; (b) Comparison of experimental and theoretical dielectric constant

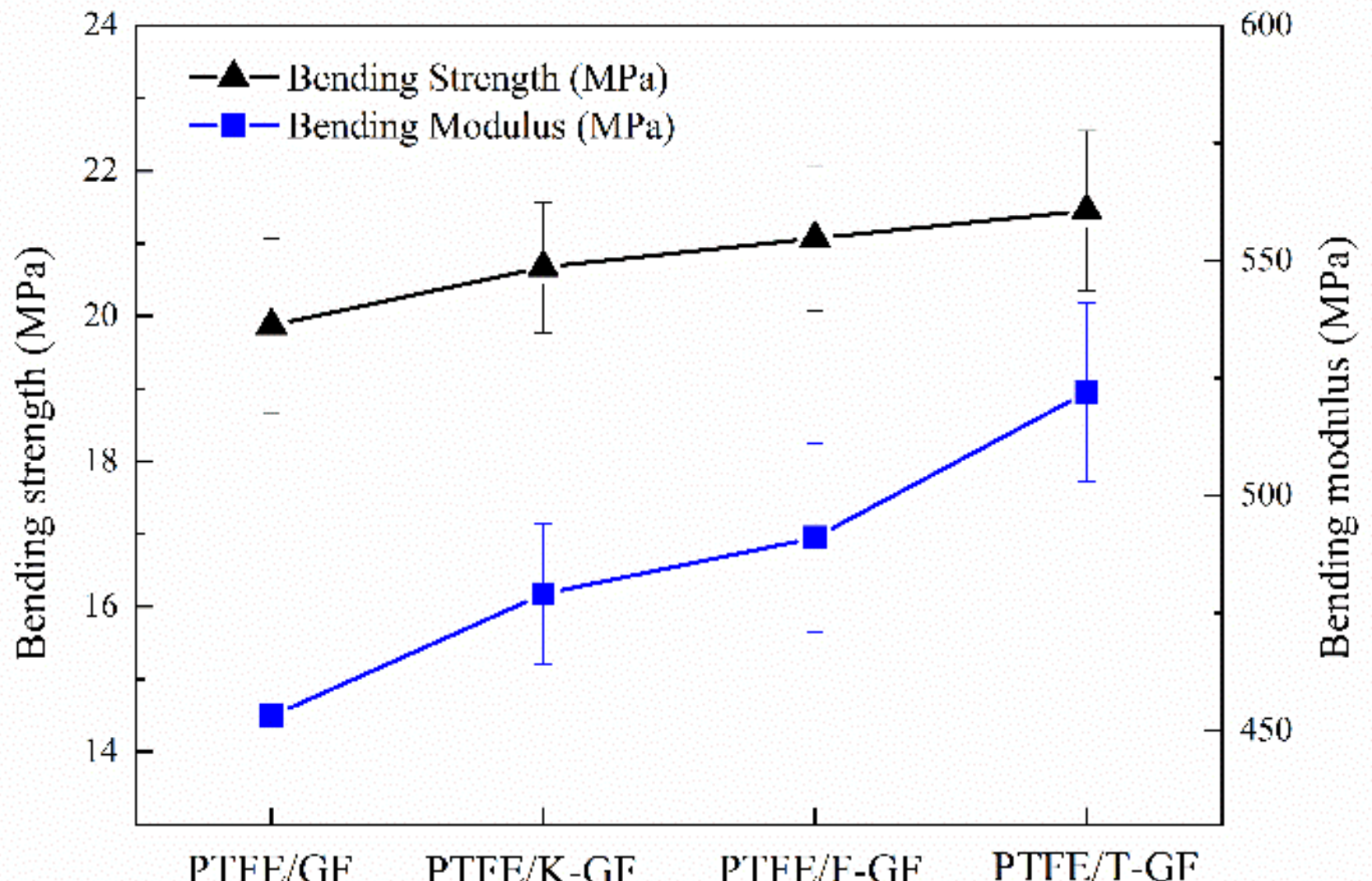

Figure 9 

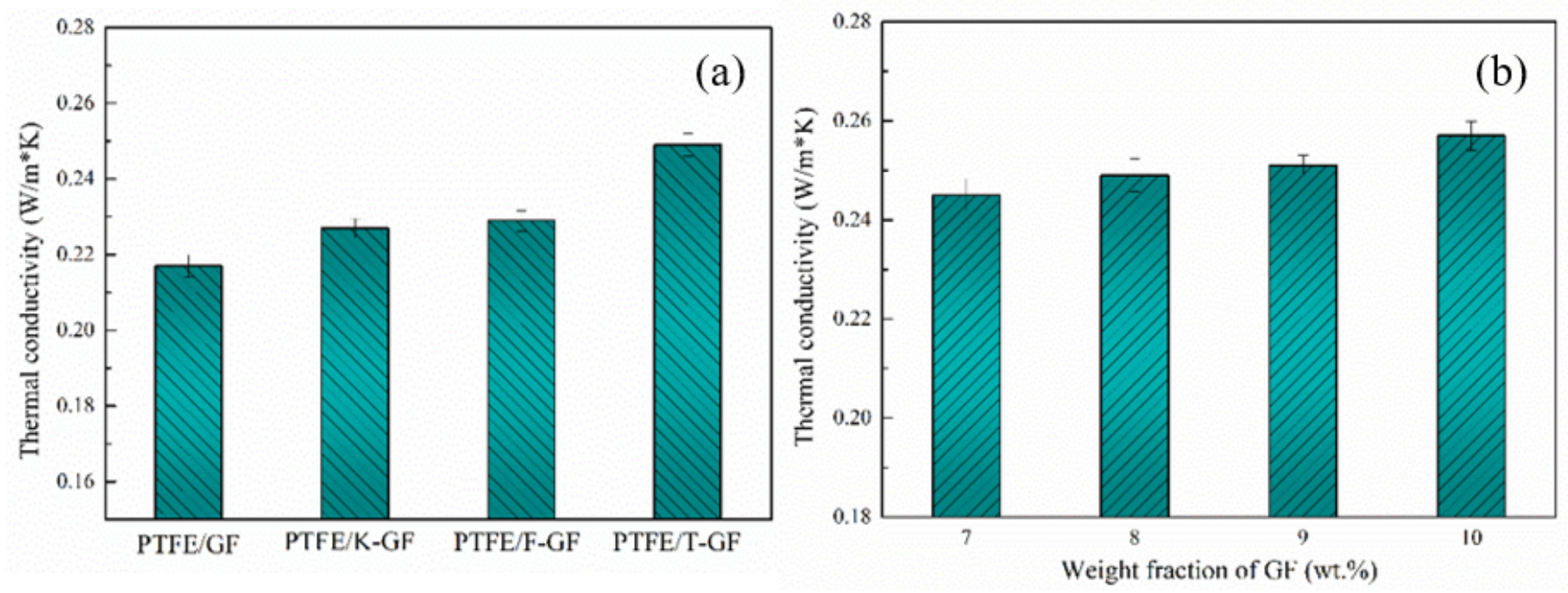

\section{Figure 10}

Thermal conductivity of (a) PTFE/GF composites with 8 wt.\% GF (b) PTFE/T-GF composites
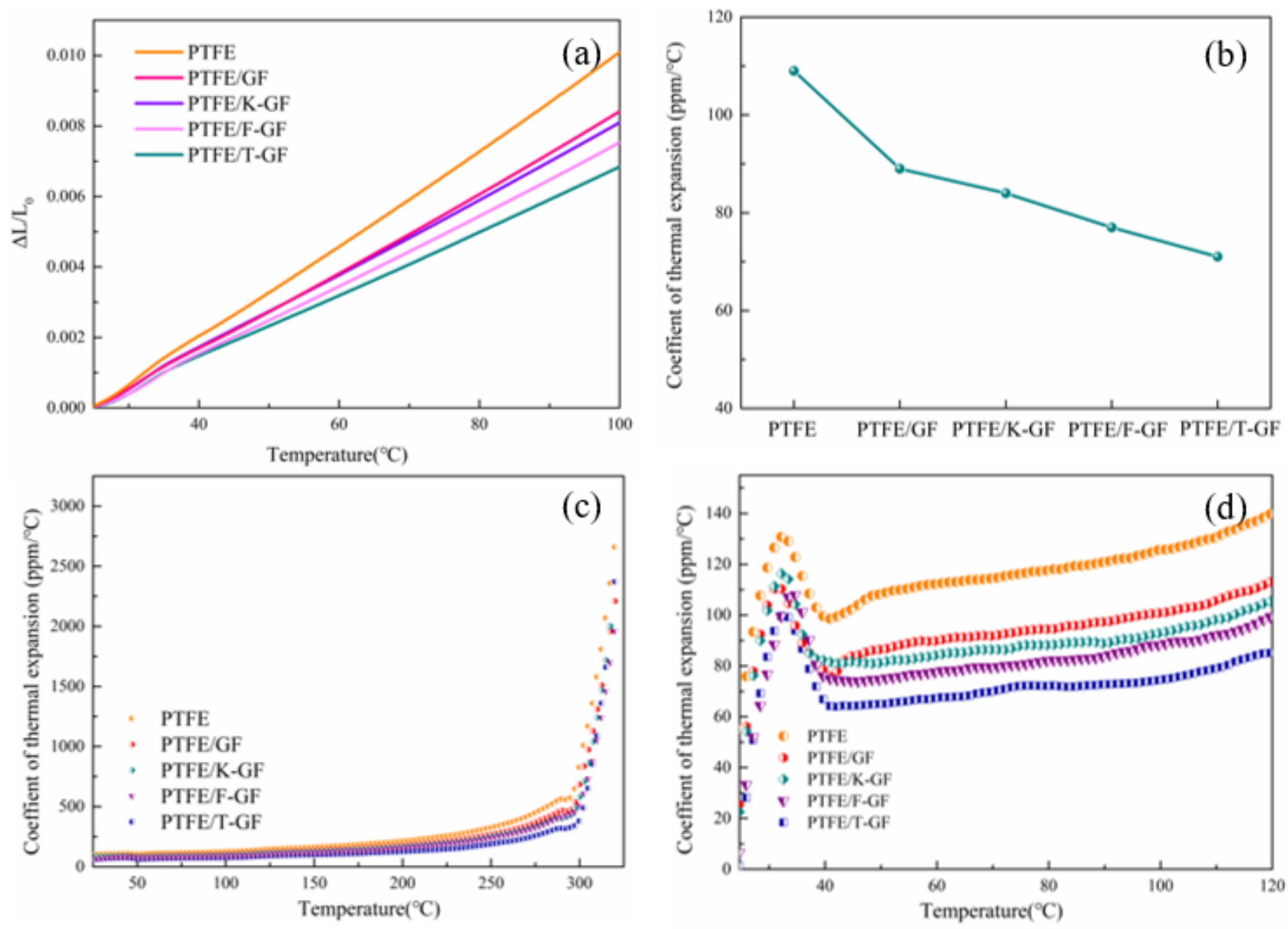


\section{Figure 11}

Dynamic mechanical curves and (b) The average CTE of PTFE and GF reinforced composites at 25-100 ${ }^{\circ} \mathrm{C}$; CTE curves of PTFE and PTFE/GF composites (c) in the range of $25-330{ }^{\circ} \mathrm{C}$ and (d) in the range of $25-120^{\circ} \mathrm{C}$ 